\title{
Research Status and Development Direction of Piezoelectric Wind Energy Harvesting Technology
}

\author{
Hongbing Wang ${ }^{1,2}$, Chunhua Sun ${ }^{1,2}$ \\ ${ }^{1}$ Department of Mechanical \& Electrical Engineering, Suzhou Vocational University, Suzhou, China \\ ${ }^{2} 3 \mathrm{C}$-Product Intelligent Manufacturing Engineering Technology Research and Development Center of Jiangsu Province, Suzhou, \\ China \\ Email: whb@jssvc.edu.cn
}

How to cite this paper: Wang, H.B. and Sun, C.H. (2019) Research Status and Development Direction of Piezoelectric Wind Energy Harvesting Technology. Journal of Power and Energy Engineering, 7, 1-10. https://doi.org/10.4236/jpee.2019.73001

Received: January 26, 2019

Accepted: March 15, 2019

Published: March 18, 2019

Copyright ( 2019 by author(s) and Scientific Research Publishing Inc. This work is licensed under the Creative Commons Attribution International License (CC BY 4.0).

http://creativecommons.org/licenses/by/4.0/ c) (i) Open Access

\begin{abstract}
In recent years, with the rapid development of large-scale distributed wireless sensor systems and micro-power devices, the disadvantages of traditional chemical battery power supply mode are becoming more and more obvious. Piezoelectric energy collector has attracted wide attention because of its simple structure, no heating, no electromagnetic interference, environmental protection and easy miniaturization. Wind energy is a reproducible resource. Wind energy harvester based on piezoelectric intelligent material can be named piezoelectric wind energy harvesting which converts wind energy into electric power and will have great application prospect. To promote the development of piezoelectric wind energy harvesting technology, research statuses on piezoelectric wind energy harvesting technology are reviewed. The existing problem and development direction about piezoelectric wind energy harvester in the future are discussed. The study will be helpful for researchers engaged in piezoelectric wind energy harvesting.
\end{abstract}

\section{Keywords}

Wind Energy, Piezoelectric Material, Energy Harvester, Reproducible Resource

\section{Introduction}

With the aggravation of global warming and the shortage of petroleum energy, it is a strategic focus to seek multi-mode, multi-source, non-polluting renewable energy for the development of science and technology in the world at present 
and in the future. Different methods of capturing energy from the surrounding environment have become the focus of recent researches. Among all kinds of energy conversion types, the efficiency of transforming mechanical energy such as pressure, impact and vibration into electric energy based on piezoelectric effect is second only to solar energy and wind energy, and it is a promising renewable energy.

Energy harvesting technology could change vibration energy into electrical energy by recycling wasted vibration energy [1]. Methods of energy conversion of vibration to electrical energy were piezoelectric, electromagnetic and electrostatic [2] [3] [4]. It had been proved that piezoelectric transducers were more suitable for vibration to electrical energy converters.

Recently, with large distributed wireless sensor systems and subminiature power devices developing quickly, traditional chemical battery could satisfy the need to some extent. But the problems such as huge volume, finite life and periodical substitution were very obvious [5].

Wind energy was a transformation of solar energy and could be taken everywhere. Traditional wind power generation was caused by the relative movement between the rotor and stator. The power supply mode had large scale and complex process, not suitable for wireless sensor systems and subminiature power devices. The principle of wind-induced vibration of piezoelectric energy harvester was the use of wind-induced vibration mechanism to convert the wind energy into electrical energy. It has the advantages of simple structure, small volume, low cost advantages, and could be used in such fields as wireless sensor systems and subminiature power devices.

For promoting the application of the piezoelectric wind energy harvesting technology, research status and development direction were discussed in the paper. Because of the wide attention of Cantilever piezoelectric energy harvester, the paper discussed especially the characteristics of the Cantilever piezoelectric energy harvester.

\section{Research Status of Piezoelectric Wind Energy Harvesting Technology}

With the development of piezoelectric wind energy harvesting technology, scholars at home and abroad have proposed a variety of piezoelectric wind energy harvesting devices, and conducted a lot of theoretical and experimental research. According to different harvesting mode piezoelectric wind energy harvesting technology can be divided into fan type and wind-induced vibration type.

\subsection{Fan Type Piezoelectric Wind Energy Harvesting Technology}

Fan-type piezoelectric energy harvesting technology utilizes the working principle of the fan, drives the turbine mechanism to rotate through the wind energy, and then causes bending deformation of piezoelectric components through the transmission mechanism. 
In 2003 two Japanese companies NEC TOKIN and Heardea developed a piezoelectric vision navigation mark [6]. The mark was a circular with diameter of about $12.3 \mathrm{~cm}$. The outer configuration was a plume shaped object and the internal configuration was a piezoelectric conversion device, a steel ball and six LED lamps. The construction of the mark was shown in Figure 1. In the highway tunnel with large traffic volume, the mark could be rotated by the wind generated from the passing cars. Because the steel ball was located in the circular channel divided by the piezoelectric conversion element, the ball would be picked up and then fall in the channel when the mark rotated. The movement of the ball would go round and begin again with the rotation of the mark. The LED lamps were lighted by the collision of the falling ball and the piezoelectric element. The device could be used in power generation. When wind speed was $3-6$ $\mathrm{m} / \mathrm{s}$, the device lighted 3 times per second, lasted more than $3 \mathrm{~ms}$ and the brightness of $4000 \mathrm{mcd}$ or more, could be seen in the $200 \mathrm{~m}$ distance.

American Texas State University developed a miniature wind driven piezoelectric power generation device [7], as shown in Figure 2. The device used a wind wheel which made 12 piezoelectric cantilever beams vibrate, and electric energy was generated in the piezoelectric layer on the twin beams. The size of the single piezoelectric cantilever beam was $60 \mathrm{~mm} \times 20 \mathrm{~mm} \times 0.6 \mathrm{~mm}$, and the size of the entire power generation device was $5.08 \mathrm{~cm} \times 11.6 \mathrm{~cm} \times 7.7 \mathrm{~cm}$. The device could generate $12 \mathrm{~mW}$ electric power.

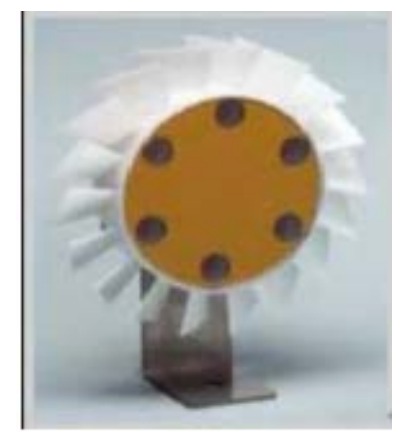

Figure 1. The piezoelectric vision navigation mark.

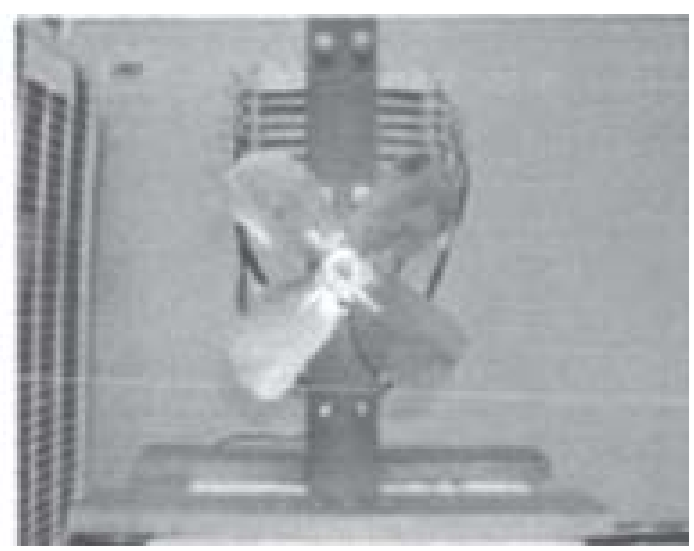

Figure 2. The wind driven piezoelectric device. 
In 2015 Hyun Jun Jung [8] designed a piezoelectric impact-based micro wind energy harvester. The harvester was shown in Figure 3. The experimental results showed that the optimized PIMWEH generated $2.8 \mathrm{mw}$.

In 2018 Luo [9] proposed the piezoelectric generator with wind cups. The generator was shown in Figure 4. The results showed that the starting wind speed of the experiment was about $3 \mathrm{~m} / \mathrm{s}$, and the optimal load of rectangular and trapezoidal piezoelectric cantilevers was $25 \mathrm{~K} \Omega$ with corresponding output power of $0.11 \mathrm{~mW}$ and $0.13 \mathrm{~mW}$.

The main characteristics of fan type piezoelectric energy harvesting technology include:

1) Fan-type piezoelectric energy harvesting technology can increase the contact area with natural wind through turbine mechanism, so that wind energy can be effectively utilized.

2) Complex turbine mechanism and transmission mechanism are installed in the fan-type piezoelectric energy capture device, which enlarges the volume of the device and is not conducive to miniaturization.

3) The efficiency of the complex transmission mechanism in the fan-type piezoelectric energy harvesting device is seriously reduced due to friction and other reasons.

\subsection{Wind-Induced Vibration Type Piezoelectric Wind Energy Harvesting Technology}

Wind-induced vibration piezoelectric energy harvesting technology refers to mechanical vibration caused by natural wind directly acting on the device, thus causing bending deformation of piezoelectric components.

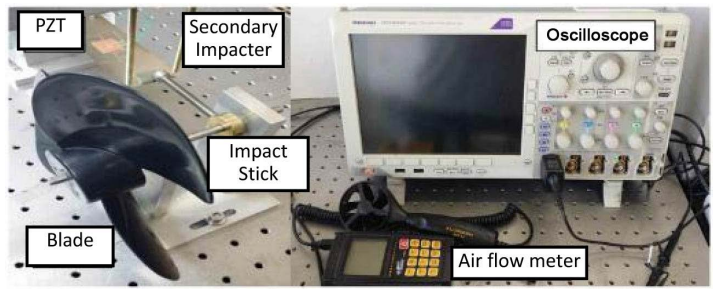

Figure 3. The impact-based micro wind energy harvester.

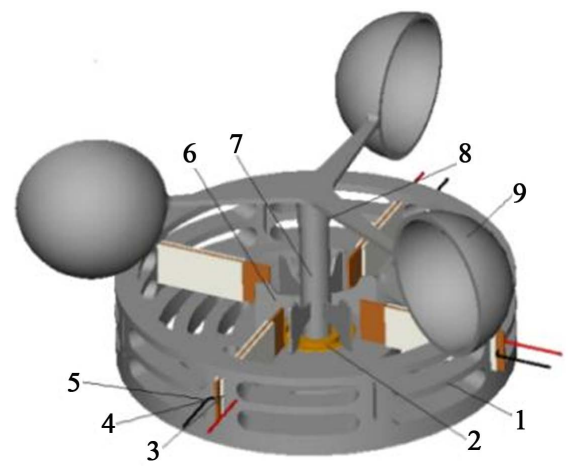

Figure 4. The piezoelectric generator with wind cup. 
In 2006 American Minnesota state university developed a piezoelectric wind generator adopting double piezoelectric film PVDF [10]. The size of the generator was $245 \mathrm{~mm} \times 178 \mathrm{~mm} \times 308 \mathrm{~mm}$. The output power was $10 \mathrm{~mW}$ and the power density was up to $4.4 \mathrm{~W} / \mathrm{cm}^{3}$ at the wind speed $19.3 \mathrm{~km} / \mathrm{h}$ and the resistive load $200 \mathrm{k} \Omega$.

In 2010 the South Korean scholars designed a portable wind generator [11], as shown in Figure 5. Fan device caused the variation of air to drive the vibration of piezoelectric cantilever beam, thus output power. The size of the generator was $250 \mathrm{~mm} \times 250 \mathrm{~mm} \times 250 \mathrm{~mm}$. The output voltage was $4.05 \mathrm{~V}$ at the wind speed $3.5 \mathrm{~m} / \mathrm{s}$ and the resonant frequency $42.7 \mathrm{~Hz}$.

In 2010 the South Korean scholars developed a small wind generator, as shown in Figure 6 [12]. The appearance of the generator looked like the word "T". The output voltage was $130 \mathrm{~V}$ and the power was $4 \mathrm{~mW}$ at the wind speed $15 \mathrm{~m} / \mathrm{s}$, the resonant frequency $6.17 \mathrm{~Hz}$ and the resistive load $4 \mathrm{M} \Omega$.

In 2010 American scholars studied a kind of portable vortex induced vibration generator [13], as shown in Figure 7. Cylindrical body produced vortex induced vibration under the action of wind, accordingly the piezoelectric ball vibrated and induced deformation, thus generated electric energy. The size of the generator was $20 \mathrm{~mm} \times 20 \mathrm{~mm} \times 255 \mathrm{~mm}$. The power was $20 \mu \mathrm{W}$ at the wind speed 1 $-3 \mathrm{~m} / \mathrm{s}$.

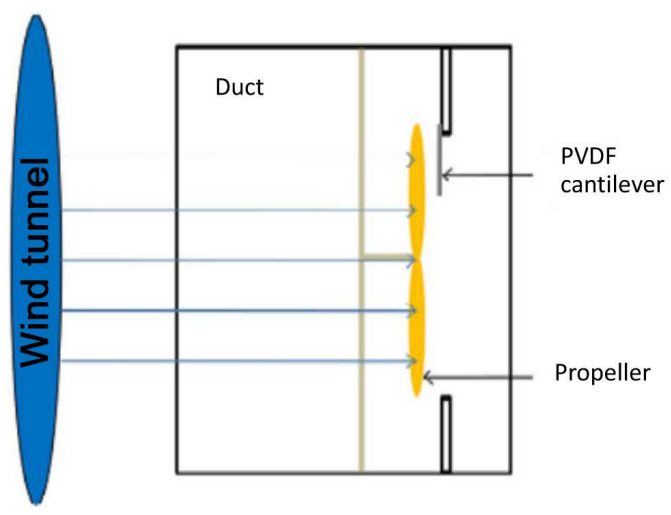

Figure 5. The portable wind generator.

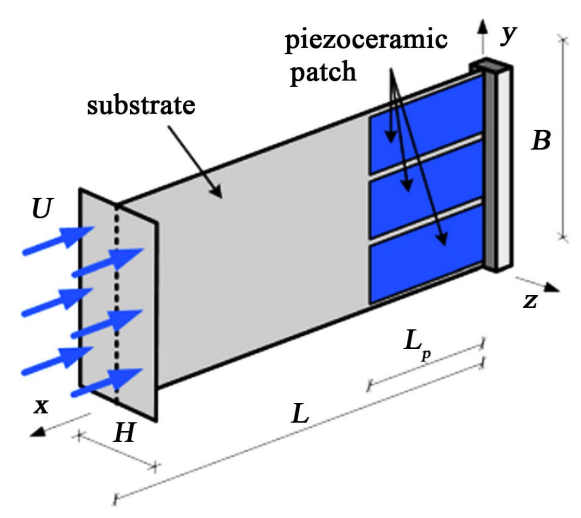

Figure 6. The small wind generator. 


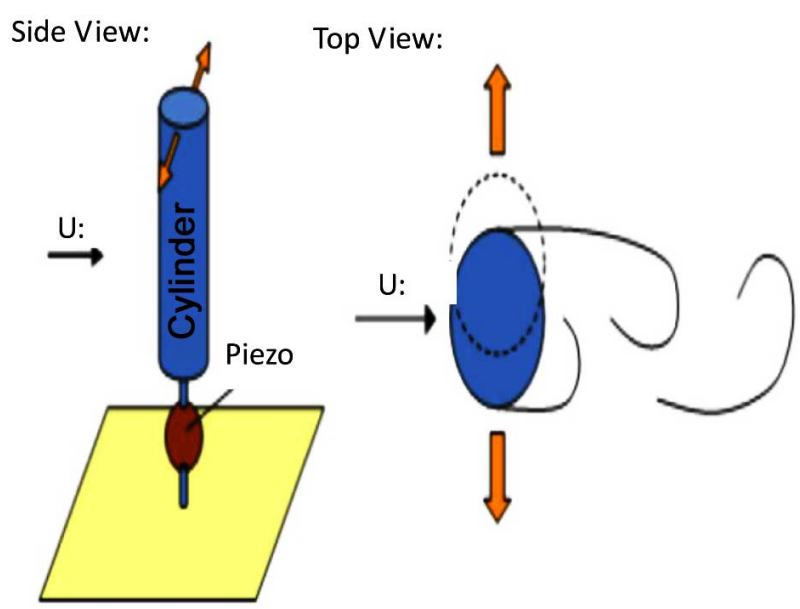

Figure 7. The portable vortex induced vibration generator.

In 2012 Singapore scholars developed a micro wind energy harvester by MEMS process [14], as shown in Figure 8. The bending of the cantilever beam structure was used to increase the force of the wind load, and then improve the electric generation efficiency. The output voltage was $18.1 \mathrm{mV}$ and the power was $3.3 \mathrm{nW}$ at the wind speed $15.6 \mathrm{~m} / \mathrm{s}$ and the resistive load $100 \mathrm{k} \Omega$.

In 2013 the University of Michigan scholars developed a turbine type wind energy harvester based on the principle of piezoelectric, as shown in Figure 9 [15]. On the piezoelectric cantilever tip and the rotating parts the permanent magnet were arranged, when the air flow drived the blade to rotate, the permanent magnet induced alternately attraction and repulsion by mutual force, which drived the vibration of the piezoelectric cantilever beam, thus wind energy was converted into electric energy. The size of the generator was $80 \mathrm{~mm} \times 80 \mathrm{~mm} \times$ $175 \mathrm{~mm}$. The generator could generate milliwatts of power from wind as slow as $2^{\circ} \mathrm{m} / \mathrm{s}$

In 2012 a resonant cavity type piezoelectric micro wind energy harvester based on the principle of piezoelectric was developed by the Chinese scholars, as shown in Figure 10 [16]. The force of wind load on the piezoelectric cantilever beam was amplified by using the resonant cavity structure, and the flexible beam Structure was used to reduce the critical wind speed of wind collector. The size of the generator was $64 \mathrm{~mm} \times 22 \mathrm{~mm} \times 14 \mathrm{~mm}$. The generator could generate $1.28 \mathrm{~mW}$ of power from the wind speed $17 \mathrm{~m} / \mathrm{s}$.

In 2018 Sibel Akkaya [17] Oy designed and tested a new piezoelectric-based low-power wind generator to generate cheap and clean energy. The generator was shown in Figure 11. The generator included an unbalanced propeller and a piezoelectric material equipped board. The average power output of $519 \mu \mathrm{w}$ was achieved at the $4.5-5 \mathrm{~m} / \mathrm{s}$ wind speed range.

In 2011, Sirohi [18] designed a mirco galloping motion piezoelectric generator which was shown in Figure 12. The generator included two piezoelectric cantilevers and one equilateral triangular prism. The test results showed that when the wind speed was $11.6 \mathrm{mph}$, the maximum output power was up to $53 \mathrm{~mW}$. 


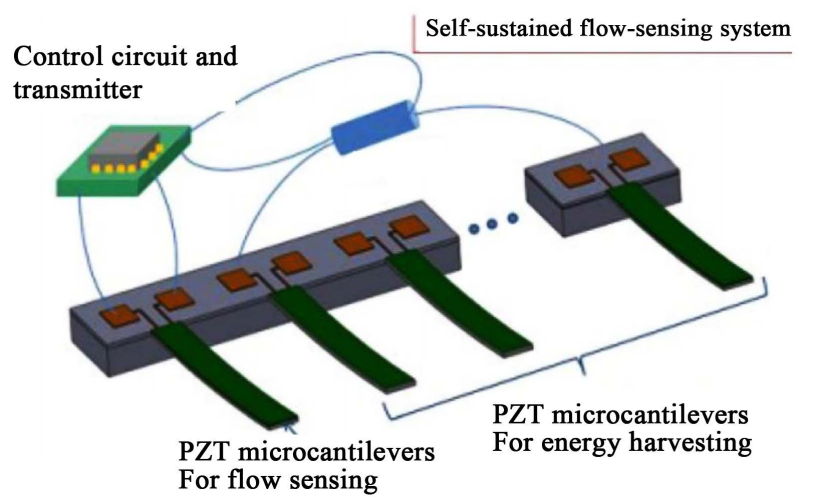

Figure 8. The micro wind energy harvester.

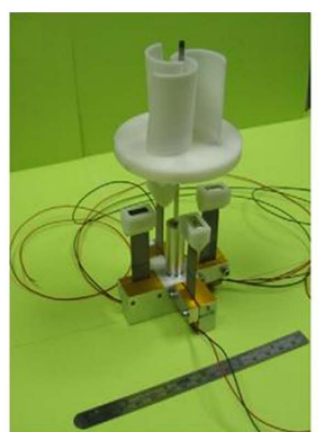

(a)

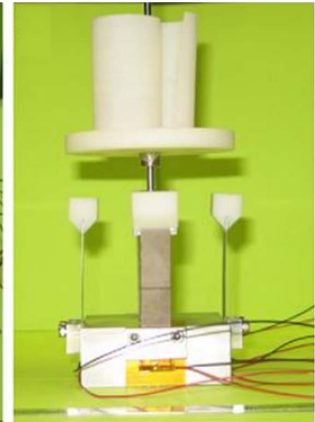

(b)

Figure 9. The turbine type wind energy harvester.

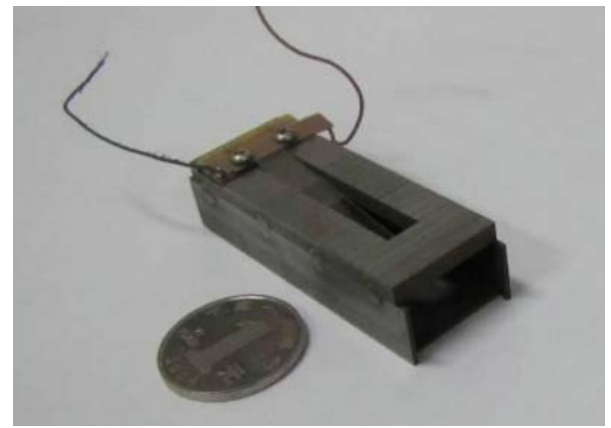

Figure 10. The small wind generator.

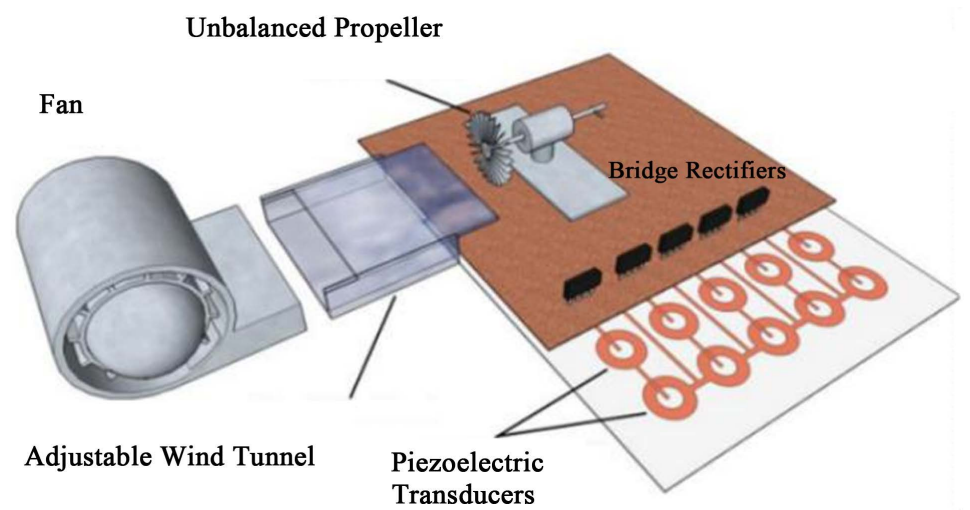

Figure 11. The piezoelectric-based low-power wind generator. 


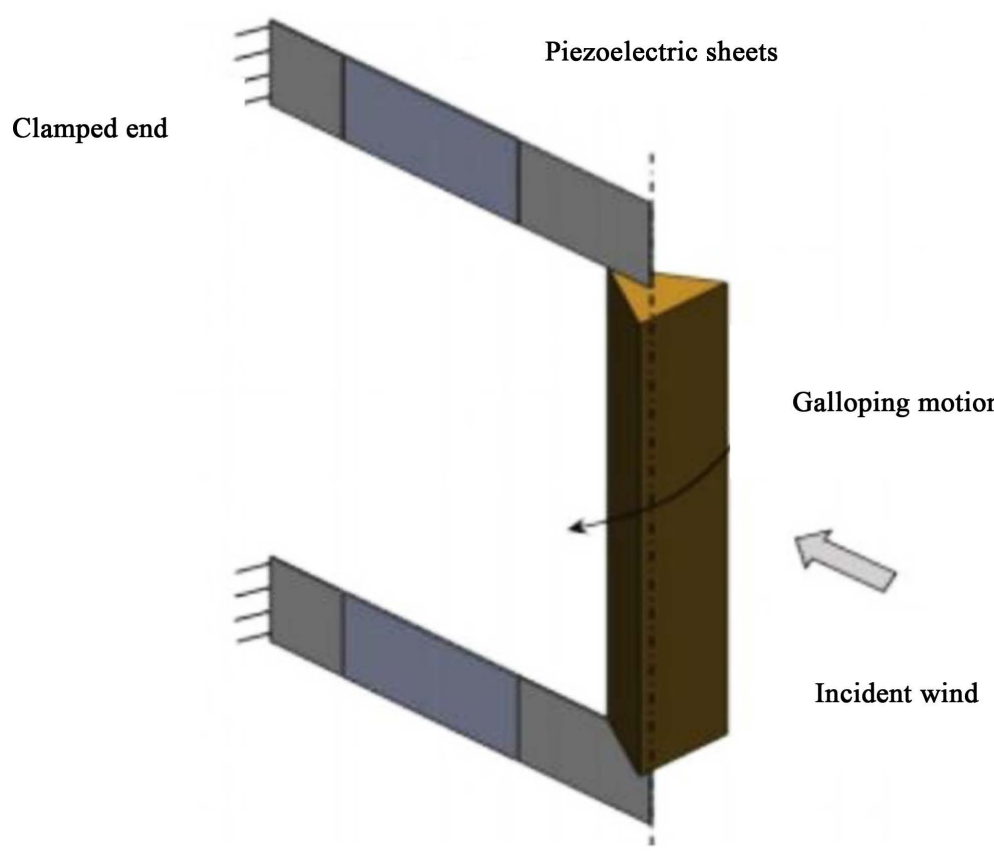

Figure 12. Mirco galloping motion piezoelectric generator.

The main characteristics of wind-induced vibration piezoelectric energy harvesting technology include:

1) The wind-induced vibration piezoelectric energy harvesting device has no intermediate transmission mechanism, which improves the efficiency of power generation.

2) Wind-induced vibration piezoelectric energy harvesting device is easy to realize miniaturization.

3) In wind-induced vibration piezoelectric energy harvesting device, the contact area between piezoelectric elements and natural wind force is small, and wind energy cannot be fully utilized.

\section{Future Development Direction}

Piezoelectric wind energy harvesting technology was related to mechanics, materials science, electrical, piezoelectric and elastic mechanics, which belonged to the intersection problem of multi discipline. The development of this technology was helpful to solve the traditional battery to the microelectronic, wireless network energy consumption problems of electronic products, which also helped to improve the micromation and integration of the modern sensor technology. The main problems existing and development direction on piezoelectric wind energy harvesting technology were shown as follows.

1) The power density of the piezoelectric wind energy harvester was low. Currently, the structure of the piezoelectric wind energy harvester was more complex, more energy loss in the conversion process, and the energy conversion efficiency was low. In order to improve the energy harvesting efficiency, researchers needed to develop new methods and new structures. 
2) Harvester reliability was poor. Piezoelectric wind energy harvester was arranged in the outdoor environment, and the working environment was relatively poor. Device was not stable and seriously hindered the further development of piezoelectric wind energy harvesting technology.

3) The sizes of the exited piezoelectric wind energy harvesters were generally larger. With the rapid development of MEMS, correspondingly micro energy device to supply energy developed quickly, and the piezoelectric wind energy harvesters of micro size were urgently needed.

4) The structure design of piezoelectric wind energy harvesters needs further study. The structure of the existing piezoelectric wind energy harvesters is relatively complex, resulting in more difficult processing, and the application of energy supply of micro-electronic products has been limited.

5) The service lives of the harvesters were low. The structure stress and strain of the harvester was changing all the time due to long time vibration. How to improve the fatigue life of piezoelectric power generation device was the urgent problem needed to solve.

\section{Acknowledgements}

This work supported by Suzhou science and technology planning project (SYG201823); Suzhou vocational university research project (SVU2018YY07); sponsored by Qing Lan Project.

\section{Conflicts of Interest}

The authors declare no conflicts of interest regarding the publication of this paper.

\section{References}

[1] Lee, J. and Choi, B. (2012) A Study on the Piezoelectric Energy Conversion System Using Motor Vibration. International Journal of Precision Engineering and Manufacturing, 13, 573-579. https://doi.org/10.1007/s12541-012-0073-8

[2] Glynne, J.P., Tudorm, J., Beeby, S.P., et al. (2004) An Electromagnetic Vibration-Powered Generator for Intelligent Sensor Systems. Sensors and Actuators A, 110, 344-349. https://doi.org/10.1016/j.sna.2003.09.045

[3] Mitcheson, P.D., Miao, P., Stark, B.H., et al. (2004) MEMS Electrostatic Micropower Generator for Low Frequency Operation. Sensors and Actuators A, 115, 523-529. https://doi.org/10.1016/j.sna.2004.04.026

[4] Choi, W.J., Jeon, Y., Jeong, J.H., et al. (2006) Energy Harvesting MEMS Device Based on Thin Film Piezoelectric Cantilevers. Journal of Electroceramics, 17, 543-548. https://doi.org/10.1007/s10832-006-6287-3

[5] Guan, M.J. and Liao, W.H. (2007) On the Efficiencies of Piezoelectric Energy Harvesting Circuits towards Storage Device Voltages. Smart Materials and Structures, 16, 498-505. https://doi.org/10.1088/0964-1726/16/2/031

[6] The Nikkei BP Agency (2003). http://china.nikkeibp.co.jp/china/news/200307/elec200307280104.html

[7] Chen, C.-T., Islam, R.A. and Priya, S. (2006) Electric Energy Generator. IEEE 
Transactions on Ultrasonics Ferroelectrics and Frequency Control, 53, 656-661. https://doi.org/10.1109/TUFFC.2006.1610576

[8] Jung, H.J., Song, Y., Hong, S.K., et al. (2015) Design and Optimization of Piezoelectric Impact-Based Micro Wind Energy Harvester for Wireless Sensor Network. Sensors and Actuators A Physical, 222, 314-321. https://doi.org/10.1016/j.sna.2014.12.010

[9] Luo, Z. (2018) Design and Research of a New Piezoelectric Wind Energy Collector. Electronic University of Science \& Technology of Hangzhou, Hangzhou.

[10] Willian, P.R. and Lian, M. (2006) Wind-Generated Electricity Using Flexible Piezoelectrics. ASME International Mechanical Engineering Congress and Exposition, Chicago, IL, 5-10 November 2006, 14050.

[11] Chang, H.K., Daejoong, K. and Jungyul, P. (2010) Design and Analysis of Portable Loadless Wind Power Source for Ubiquitous Sensor Network. 2nd International Conference on Computer and Automation Engineering, Singapore, 26-28 February 2010, 93-96.

[12] Kwon, S.D. (2010) A T-Shaped Piezoelectric Cantilever for Fluid Energy Harvesting. Applied Physics Letters, 97, 1-3. https://doi.org/10.1063/1.3503609

[13] William, B.H. (2010) Piezoelectric Energy Harvesting: Vortex Induced Vibrations in Plants, Soap Films, and Arrays of Cylinders. Georgia Institute of Technology.

[14] Liu, H.C., Zhang, S.S., Kathiresan, R., Kobayashi, T., et al. (2012) Development of Piezoelectric Microcantilever Flow Sensor with Wind-Driven Energy Harvesting Capability. Applied Physics Letters, 100, Article ID: 223905. https://doi.org/10.1063/1.4723846

[15] Amin, K.M., Farmer, J.R. and Inman, D.J. (2013) Parametrically Excited Nonlinear Piezoelectric Compact Wind Turbine. Renewable Energy, 50, 977-987. https://doi.org/10.1016/j.renene.2012.07.037

[16] Du, Z. and He, X. (2012) Micro Piezoelectric Wind Energy Harvester with a Resonant Cavity. Chinese Journal of Sensors and Actuators, 25, 748-750.

[17] Akkayaoy, S. and Ozdemir, A.E. (2018) Based Low-Power Wind Generator Design and Testing. Arabian Journal for Science and Engineering, 43, 2759-2767. https://doi.org/10.1007/s13369-017-2799-1

[18] Sirohi, J. and Mahadik, R. (2011) Piezoelectric Wind Energy Harvester for Low-Power Sensors. Journal of Intelligent Material Systems and Structures, 22, 2215-2228. https://doi.org/10.1177/1045389X11428366 